\title{
评 述＼cjkstart农药化学专刊
}

\section{$N$-烷基吡唑-5-甲酰胺类杀虫杀螨剂的研究进展}

\author{
宋红健 ${ }^{(1)}$, 刘玉秀(1)，汪清民(1)* \\ (1) 元素有机化学国家重点实验室, 南开大学元素有机化学研究所, 天津 300071 \\ (2) 化学化工协同创新中心(天津), 天津 300071 \\ *通讯作者, E-mail: wangqm@nankai.edu.cn.
}

收稿日期: 2016-04-14; 接受日期: 2016-05-13; 网络版发表日期: 2016-09-26

国家自然科学基金(编号: 21132003, 21372131, 21602117)和高等学校博士学科点专项科研基金(编号: 20130031110017)资助项目

\begin{abstract}
摘要 $\quad N$-烷基吡唑-5-甲酰胺类杀虫、杀螨剂因具有高效、低毒和作用机理独特等特点, 在农药研究领域占有 重要地位. 这类化合物的合成和生物活性研究一直是农药研究领域的一个热点. 本文按结构对其进行分类, 综 述了 $N$-烷基吡唑-5-甲酰胺类杀虫、杀螨剂的研究进展, 并对其结构与活性的关系进行了简单概括.
\end{abstract}

关键词 $N$-烷基吡唑-5-甲酰胺, 吡螨胺, 唑虫酰胺, 杀虫活性, 杀螨活性

\section{1 引言}

农业害虫和害螨对果树、蔬菜、茶树等作物的危 害很大，它们易于对杀虫、杀螨剂产生抗性，农业生 产中频繁单一地使用杀虫、杀螨剂更加速了抗性的产 生. 例如, 20 世纪 90 年代由德国拜耳公司开发的新烟 碱类杀虫剂吡虫啉, 作为一种高效的杀虫剂对刺吸 式口器害虫具有很好的防治效果, 但是由于部分地 区长时间大量的使用使得害虫对其产生了抗性，防 治效果明显下降 ${ }^{[1,2]}$. 为了延缓抗性的产生, 延长药 剂的使用寿命, 可采用轮用和混用这两种用药策略. 轮用就是在植物的某一生育期内, 交替使用作用机 制完全不同的农药; 混用是将两种或两种以上的农 药按一定比例混合在一起, 经过加工制成一定剂型 的混剂即复配农药. 此外, 为了应对所出现的抗性问 题，则需要持续创制新结构和新作用机制的农药.

目前已开发出来的具有新颖结构和作用机制的
杀虫、杀螨剂主要有季酮酸类、拟除虫菊酯类、新烟 碱类、吡唑类、嘧啶类、吡咯类以及天然产物类等 ${ }^{[3]}$. 其中, 吡唑类农药是一类重要的杂环类化合物, 广泛 用于杀虫、杀螨、杀菌和除草等多个领域 ${ }^{[4]}$. 目前已 经商品化的吡唑类杀虫、杀螨剂有很多种, 代表性的 品种有氟虫腈(fipronil)、吡螨胺(tebufenpyrad)、唑虫 酰胺(tolfenpyrad)、唑螨酯(fenpyroximate)、氯虫苯甲 酰胺(chlorantraniliprole)和吡唑硫磷(pyraclofos)等(图 1). 其中, $N$-烷基吡唑-5-甲酰胺类农药以其作用机理 独特、安全高效、无交互抗性、有效用量小等优点而 备受关注，其代表性的品种有吡螨胺和唑虫酰胺.

吡螨胺是由原日本三菱化学公司(其农药部分现 属日本农药公司)1987年发现的具有吡唑-5-甲酰胺结 构的杀螨剂, 1993 年在日本进行农药登记, 与美国氰 胺公司、山道士公司共同开发并以商品名 Masai 或 Pyranica 上市, 并在 10 多个国家销售. 吡螨胺防治各 种螨类、半翅目和同翅目害虫具有卓越的效果. 吡螨

引用格式: 宋红健, 刘玉秀, 汪清民. $N$-烷基吡唑-5-甲酰胺类杀虫杀螨剂的研究进展. 中国科学: 化学, 2016, 46: 1143-1154

Song H, Liu Y, Wang Q. Recent advances in insecticides/acaricides of N-alkylpyrazole-5-formamides. Sci Sin Chim, 2016, 46: 1143-1154, doi: $10.1360 / \mathrm{N} 032016-00080$ 


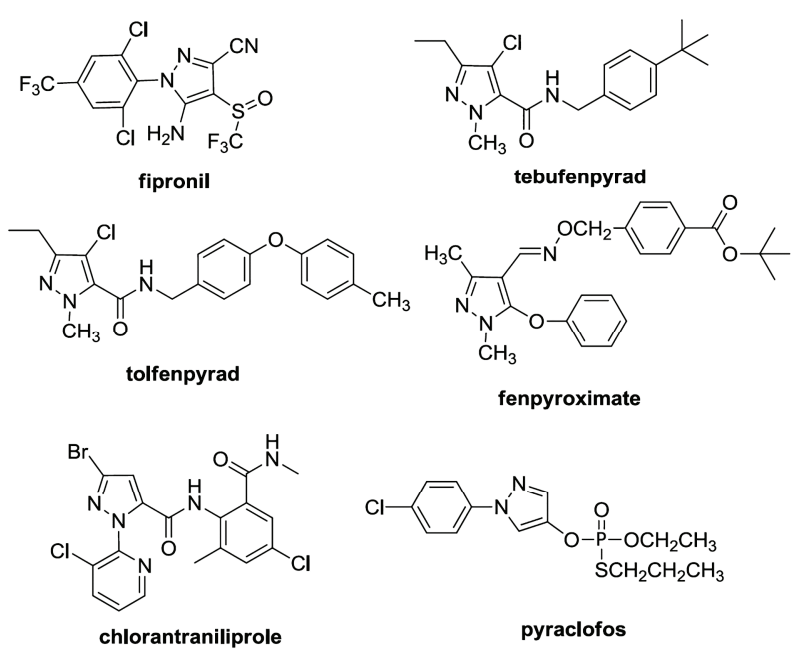

图 1 部分商品化的吡唑类杀虫、杀螨剂

胺的杀螨杀虫的作用机制是抑制线粒体的呼吸作用, 阻止电子由 NADH (还原型烟酰胺腺嘌呤二核苷酸) 通过复合物 $\mathrm{I}$ (辅酶 $\mathrm{Q}$ 还原酶) 向辅酶 $\mathrm{Q}$ 的传递, 从而 使昆虫不能提供和咜存能量.

唑虫酰胺是原日本三菱化学公司开发的新型吡 唑杂环类杀虫、杀螨剂, 其作用机理与吡螨胺基本相 同, 被称为线粒体电子传达复合体阻碍剂. 唑虫酰胺 的杀虫谱很广, 对各种鳞翅目、半翅目、鞘翅目、膜 翅目、双翅目、蓟马目及螨类均有效, 对半翅目中的 蚜虫、缨翅目的蓟马类具有种间差异小的特点, 具有 触杀作用, 对鳞翅目幼虫小菜蛾、缨翅目害虫蓟马有 特效。该药剂还具有杀卵、抑食、抑制产卵及杀菌 作用.

\section{$2 N$-烷基吡唑-5-甲酰胺类杀虫、杀螨剂的研 究进展}

1991 年, 日本三菱化学公司的 Okada 等 ${ }^{[5]}$ 报道了 具有如图 2 所示结构的吡唑-5-羰基结构的化合物.

通过对吡唑环上的取代基 $R^{1} 、 R^{2}$ 和 $R^{3}$ ，苯环上的 取代基 $\mathrm{R}^{4}$ 及其在苯环上的位置以及 $\mathrm{X} 、 \mathrm{Y}$ 的研究最终 发现, 具有表 1 所示结构的化合物对二斑叶螨 (Tetranychus urticae) 和柑橘全爪螨(Panonychus citri) 具有很好的杀螨和杀螨卵活性. 其中, 当 $\mathrm{R}^{2}$ 为乙基、 $\mathrm{R}^{3}$ 为氯时表现更加优异, 即为商品化品种乙螨坐.

在 Okada 等 ${ }^{[5]}$ 报道 $N$-烷基吡唑-5-甲酰胺类化合物 表现出优异的杀虫、杀螨活性前后, 对于具有此类结
构的吡唑类化合物的研究成为了一个热点, 总结文献 中报道的具有生物活性的吡唑类化合物，按结构可分 为以下 3 类(图 3): (1) 对 $\mathrm{A}$ 部分(吡唑环部分)取代基的 修饰; (2) 对 B 部分(酰胺键部分)的修饰; (3) 对 C 部分 (苯环部分)的修饰.

\section{1 对 $\mathrm{A}$ 部分的修饰}

对 $\mathrm{A}$ 部分(吡唑环部分)的修饰主要是对吡唑环 3 位和 4-位的修饰, 主要包括以下 3 个方面: (1) 3-, 4位成环; (2) 将 4-位的取代基 $\mathrm{R}^{3}$ 换成烷氧基、酯基、 不饱和取代基等; (3) 用其他杂环代替吡唑环.

\subsubsection{3-, 4-位成环}

1989 年, Okada 等 ${ }^{[6]}$ 合成了一系列 3-, 4-位成五元脂<smiles>[X][Y](=C([X])c1c([R])c([R])nn1[R1])c1ccc([R])cc1</smiles>

图 2 吡唑-5-羰基化合物

表 1 高活性化合物对二斑叶螨和柑橘全爪螨成螨和螨卵的 $\mathrm{LC}_{50}$ 值

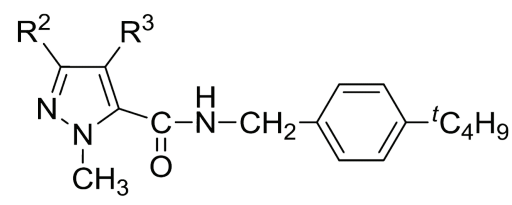

\begin{tabular}{|c|c|c|c|c|c|c|}
\hline \multirow{3}{*}{ 编号 } & \multirow{3}{*}{$\mathrm{R}^{2}$} & \multirow{3}{*}{$\mathrm{R}^{3}$} & \multicolumn{4}{|c|}{$\mathrm{LC}_{50}(\mathrm{ppm})$} \\
\hline & & & \multicolumn{2}{|c|}{ 二斑叶螨 } & \multicolumn{2}{|c|}{ 柑橘全爪螨 } \\
\hline & & & 成螨 & 螨卵 & 成螨 & 螨卵 \\
\hline 1 & $\mathrm{CH}_{3}$ & $\mathrm{Cl}$ & 7.7 & 2.4 & 3.1 & 9.9 \\
\hline 2 & $\mathrm{CH}_{3}$ & $\mathrm{Br}$ & 9.8 & 3.0 & 4.0 & 17 \\
\hline 3 & $\mathrm{C}_{2} \mathrm{H}_{5}$ & $\mathrm{Cl}$ & 3.7 & 0.96 & 0.50 & 3.9 \\
\hline 4 & $\mathrm{C}_{2} \mathrm{H}_{5}$ & $\mathrm{Br}$ & 7.1 & 1.4 & 0.59 & 10 \\
\hline 5 & $\mathrm{CH}_{3}$ & $\mathrm{CH}_{3}$ & 7.5 & 8.8 & 6.1 & 36 \\
\hline
\end{tabular}

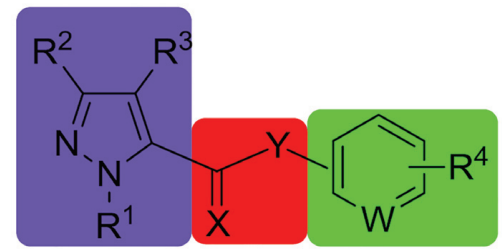

A B C

图 $3 N$-烷基吡唑-5-甲酰胺的骨架结构(网络版彩图) 
肪环的化合物(图 4). 化合物 6 9 在 $500 \mathrm{ppm}$ 下对二 斑叶螨、褐飞闽、小菜蛾有 $100 \%$ 的致死率. 它们具 有共同的特点: 苯环对位都有空间位阻比较大的疏 水性取代基. 而苯环对位含有烷氧基、烷胺基、苯氧 基和酰胺取代基的化合物时，化合物 10 13 在 500 ppm 下对褐飞闽和小菜蛾表现出杀虫活性. 这说明苯环 对位的取代基对于化合物是否具有杀螨活性起到关 键作用, 一般苯环对位具有空间位阻较大的烷基取 代基的化合物表现出较好的杀螨活性.

1992 年, Okada 等 ${ }^{[7]}$ 报道了吡唑环 3-, 4-位具有环 状结构的吡唑-5-羰基结构的化合物(图 5). 主要研究 环的大小及环上取代基对活性的影响.

通过表 2 中所列化合物可以发现，吡唑环 3-, 4-位 成五元环的活性要高于成六元环的活性, 且 $R^{2}$ 和 $R^{3}$ 分别为甲基和氢的化合物的活性最好. 经过篮选所得 的高活性化合物 15 和吡螨胺相比, 对成螨的杀螨活性 提高，而对螨卵的杀卵活性下降.

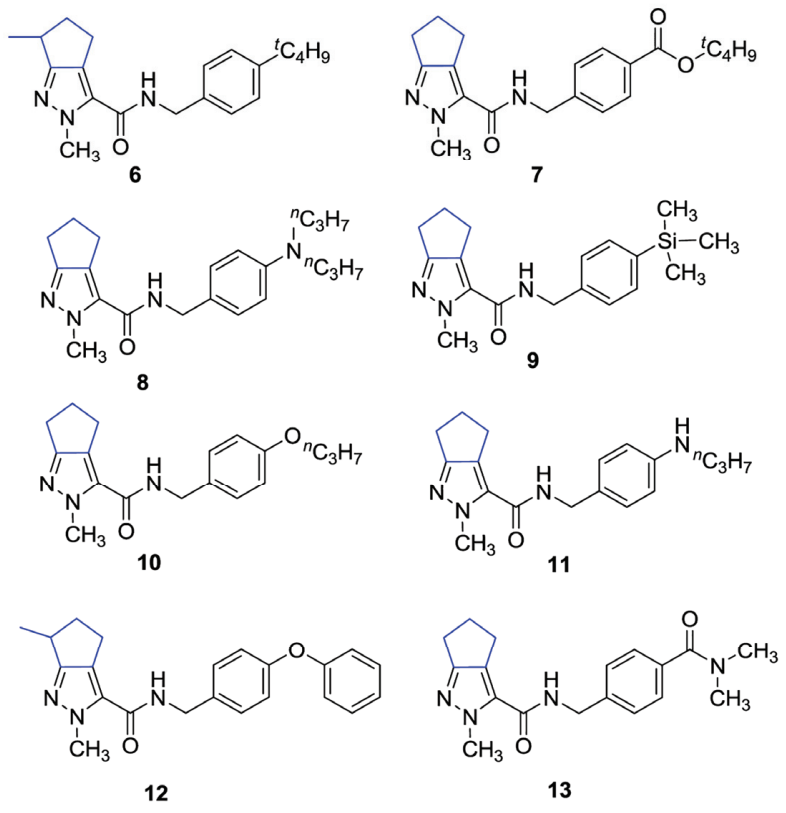

图 4 3-, 4-位成五元脂肪环的化合物(网络版彩图)

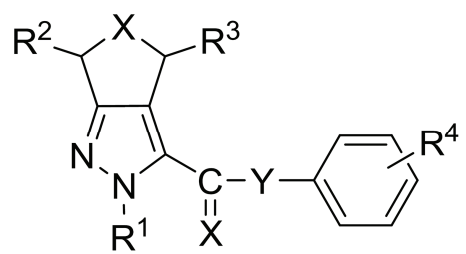

图 5 3-, 4-位具有环状结构的吡唑-5-羊基结构的化合物
表 2 取代基 $\mathrm{R}^{2} 、 \mathrm{R}^{3}$ 和 3-, 4-位成环大小对活性的影响 a)

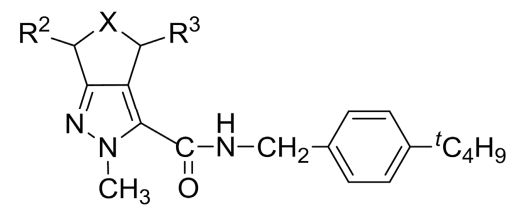

\begin{tabular}{|c|c|c|c|c|c|c|c|}
\hline \multirow{2}{*}{ No. } & \multirow{2}{*}{$\mathrm{R}^{2}$} & \multirow{2}{*}{$X$} & \multirow{2}{*}{$\mathrm{R}^{3}$} & \multicolumn{4}{|c|}{$\mathrm{LC}_{50}(\mathrm{ppm})$} \\
\hline & & & & 200 & 50 & 12.5 & 3.1 \\
\hline 14 & $\mathrm{H}$ & $\mathrm{CH}_{2}$ & $\mathrm{H}$ & 3 & 3 & 3 & 2 \\
\hline 15 & $\mathrm{CH}_{3}$ & $\mathrm{CH}_{2}$ & $\mathrm{H}$ & 3 & 3 & 3 & 3 \\
\hline 16 & $\mathrm{C}_{2} \mathrm{H}_{5}$ & $\mathrm{CH}_{2}$ & $\mathrm{H}$ & 0 & 0 & 0 & 0 \\
\hline 17 & $\mathrm{CH}_{3}$ & $\mathrm{CH}_{2}$ & $\mathrm{CH}_{3}$ & 1 & 0 & 0 & 0 \\
\hline 18 & $\mathrm{CH}_{3}$ & $\mathrm{CH}_{2}$ & $\mathrm{CH}_{3}$ & 1 & 0 & 0 & 0 \\
\hline 19 & $\mathrm{H}$ & $\mathrm{CH}_{2} \mathrm{CH}_{2}$ & $\mathrm{H}$ & 2 & 1 & 0 & 0 \\
\hline 20 & $\mathrm{CH}_{3}$ & $\mathrm{CH}_{2} \mathrm{CH}_{2}$ & $\mathrm{H}$ & 0 & 0 & 0 & 0 \\
\hline
\end{tabular}

a) 活性水平级别用 $0 \sim 3$ 表示, 分别对应对二斑叶螨的杀螨 率为 0 29\%, 30\% 79\%, 80\% 99\% 和 $100 \%$.

1994 年, Farooq 等 $^{[8]}$ 报道了一系列具有吲唑结构 的化合物(图 6). 化合物 21 24 在 $400 \mathrm{ppm}$ 下对褐飞 䖝、烟叶蛾、玉米根蚜蝇和棉铃象甲有 $100 \%$ 的杀虫 活性.

1998 年, 三菱化学公司的 Kyomura 等 ${ }^{[9]}$ 也报道 了类似的化合物(图 7), 化合物 $\mathbf{2 5 ~ 2 9}$ 在 $10 \mathrm{ppm}$ 下大 部分化合物对马铃薯晚疫病菌有 $100 \%$ 的抑制活性.

\subsection{2 吡唑环 4-位的取代基为烷氧基、酯基、不饱 和取代基等}

1990 年, Okada 等 ${ }^{[10]}$ 报道了吡唑环 4-位为烷氧基 的化合物(图 8), 化合物 30 32 在 $12.5 \mathrm{ppm}$ 下对二斑
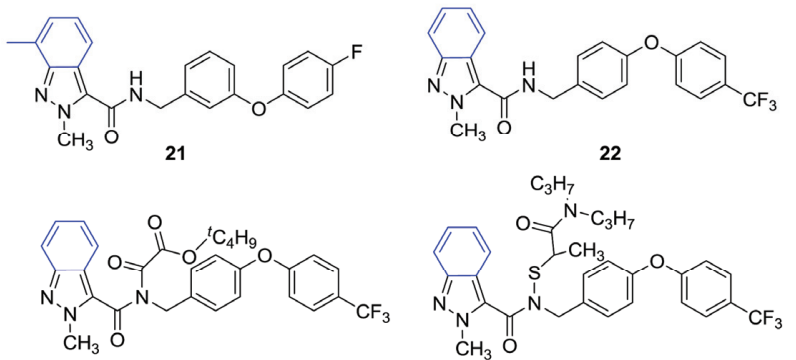

23

24

图 6 含有吲唑结构的化合物(网络版彩图)

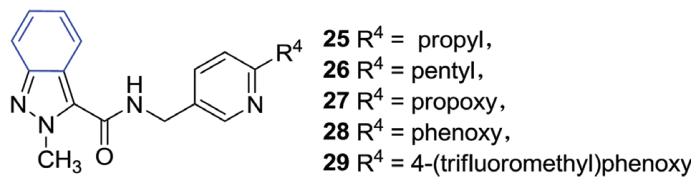

图 7 含有吲唑结构的化合物(网络版彩图) 
<smiles>CCc1nn(C)c(C(=O)NCc2ccc(C(C)(C)C)cc2)c1OC</smiles>

图 8 吡唑环 4-位为烷氧基的化合物(网络版彩图)

叶螨有 $100 \%$ 的杀螨活性以及在 $500 \mathrm{ppm}$ 对小菜蛾具 有 $100 \%$ 的杀虫活性.

1995 年, 三菱化学公司的 Tanaka 等 ${ }^{[11]}$ 报道了吡 唑环 4-位为烷氧基, $\mathrm{C}$ 部分为吡啶结构的化合物(图 9). 化合物 33 36 在 $500 \mathrm{ppm}$ 下对小菜蛾、稻飞闽、小豆 象虫以及二斑叶螨的卵和成螨具有 $100 \%$ 活性, 在 $200 \mathrm{ppm}$ 对稻瘟病和马铃薯晚疫病有 $100 \%$ 抑制性.

1998 年, 三菱化学公司的 Okimura 等 ${ }^{[12]}$ 报道了 吡唑环 4-位为烷氧基, $\mathrm{C}$ 部分为吡啶苯醚类化合物(图 10). 具有该类结构的化合物表现出杀虫、杀螨以及抑 菌活性. 化合物 37 39 在 $500 \mathrm{ppm}$ 下对稻飞䖝和小菜 蛾具有 $100 \%$ 的杀虫活性, 对二斑叶螨的成螨和卵有 $100 \%$ 的活性, 在 $250 \mathrm{ppm}$ 下对稻瘟病和马铃薯晚疫 病具有 $100 \%$ 的抑制率.

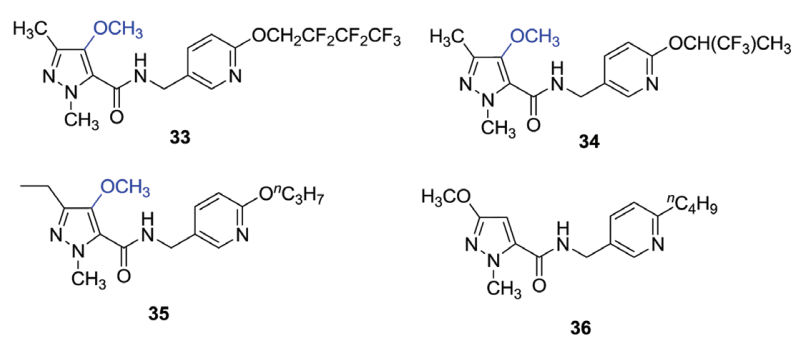

图 9 吡唑环 4-位为烷氧基, $\mathrm{C}$ 部分为吡啶结构的化合物(网 络版彩图)

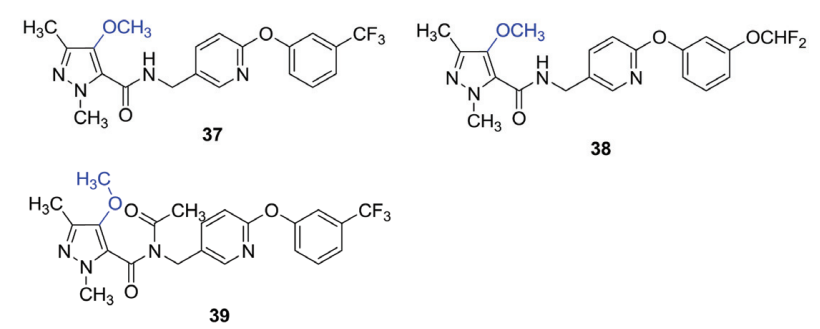

图 10 吡唑环 4-位为烷氧基, $\mathrm{C}$ 部分为吡啶苯醚类化合物 (网络版彩图)
1999 年, 日本三菱化学公司的 Kano 等 ${ }^{[13]}$ 合成了 一系列吡唑环 4-位为乙炔基和乙烯基这两种不饱和 取代基的化合物(图 11). 化合物 40 43 在 500 ppm 下 对稻飞䖝和小菜蛾具有 $100 \%$ 的杀虫活性, 对二斑叶 螨的成螨和卵有 $100 \%$ 的杀螨和杀螨卵活性, 在 $250 \mathrm{ppm}$ 下对稻瘟病、小麦锈病以及马铃薯晚疫病具有很好的 抑制活性.

\subsection{3 用其他杂环代替吡唑环}

1994 年, Okada 等 ${ }^{[14]}$ 合成了一系列如图 12 所示 的 $\mathrm{A}$ 部分为吡唑和噻唑类五元杂环化合物. 研究发 现, 如图 12 所示的五元环羰基的 $\beta$-位为甲基取代的 碳原子或甲基取代的氮原子, 以及 $\gamma$-位为不带取代 基氮原子时化合物具有较高的杀螨活性. 例如, 化合 物 44 在 $50 \mathrm{ppm}$ 下对二斑叶螨成螨有 $100 \%$ 的杀螨活 性, 而化合物 $\mathbf{4 5}$ 在 $500 \mathrm{ppm}$ 下未表现出对二斑叶螨 成螨的杀螨活性. 也就是说, 化合物 $\mathrm{A}$ 部分保持吡唑 环时杀螨活性能够保持, 改为噻唑环或其他环活性 下降.

\section{2 对 $B$ 部分的修饰}

\subsubsection{N 上有酰基取代基}

1991 年, 日本三菱化学公司的 Tanaka 等 ${ }^{[15]}$ 报道

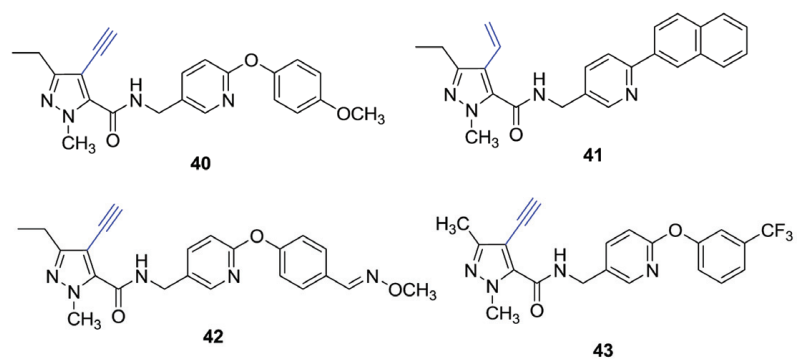

图 11 吡唑环 4-位为乙炔基和乙烯基的化合物(网络版彩图)
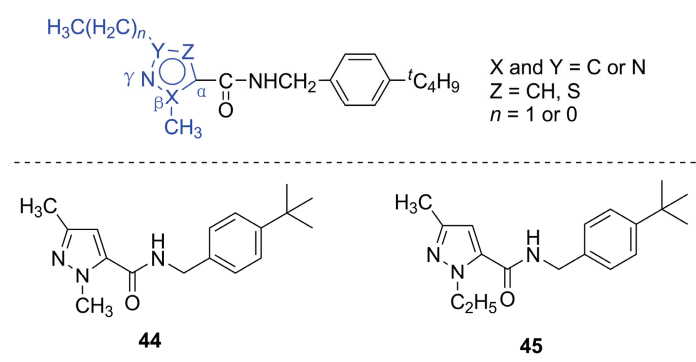

图 $12 \mathrm{~A}$ 部分为吡唑和噻唑类五元杂环化合物及高活性化 合物 44 和 45 (网络版彩图) 
了酰胺键部分 $\mathrm{N}$ 上有酰基取代, $\mathrm{C}$ 环部分为吡啶结构 的一类化合物(图 13). 化合物 $\mathbf{4 6}$ 和 $\mathbf{4 7}$ 在 $3.1 \mathrm{ppm}$ 下 对二斑叶螨有 $100 \%$ 的杀螨活性, 在 $500 \mathrm{ppm}$ 具有 $100 \%$ 的杀虫活性. 化合物 $\mathbf{4 8}$ 在 $3.1 \mathrm{ppm}$ 下对二斑叶 螨有 $100 \%$ 的杀螨活性.

2003 年, 三菱化学公司的 Shiga 等 ${ }^{[16]}$ 合成了一系 列 $\mathrm{N}$ 上含有酰基, $\mathrm{C}$ 环部分为二苯醚结构的化合物(图 14，15). 他们研究吡唑环 3 -位的取代基 $\mathrm{R}^{1}$ 对斜纹夜 蛾(Spodoptera litura) 杀虫活性的影响, 生物活性测试 结果表明, 当 $\mathrm{R}^{1}$ 为乙基时活性最好, 在 $3.1 \mathrm{ppm}$ 有 $80 \%$ 的杀虫率. 活性顺序为: 乙基 $>$ 丙基 $>$ 环丙基 $>$ 仲 丁基 $>$ 甲基、异丙基 $>$ 异丁基 $>$ 叔丁基、苯基. 其中甲 氧基、乙氧基和异丙氧基取代的化合物在 $50 \mathrm{ppm}$ 下 对斜纹夜蛾也表现出活性, 但明显低于含有乙基的 化合物.

他们又研究了酰胺氮上的取代基对化合物杀虫活 性的影响(图 15). 通过生物活性测试发现, 酰胺氮上 有酰基取代时, 活性要优于没有取代的化合物. 其中, 当 $\mathrm{R}^{3}$ 为乙酰基和异丁酰基时活性最好, 在 $3.1 \mathrm{ppm}$ 下 对斜纹夜蛾(Spodoptera litura) 的杀虫率大于 $80 \%$.

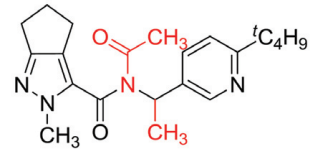

46<smiles>CCc1ccc(CN(C(C)=O)C(=O)c2c(Cl)c(CC)nn2C)cn1</smiles>

48

图 13 酰胺键部分 $\mathrm{N}$ 上有酰基取代, $\mathrm{C}$ 环部分为吡啶结构的 化合物(网络版彩图)<smiles>[R]c1cc(C(=O)N(Cc2ccc(Oc3ccc(C#N)cc3)cc2)C(C)=O)n(C)n1</smiles>

图 $14 \mathrm{~N}$ 上含有乙酰基的化合物(网络版彩图)

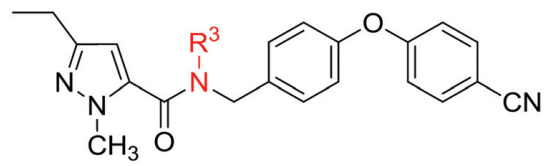

图 $15 \mathrm{~N}$ 上含有不同酰基的化合物(网络版彩图)
2001 年, 日本三菱化学株式会社的 Okada 等 ${ }^{[17,18]}$ 报道了 $\mathrm{N}$ 上含有酰基, $\mathrm{C}$ 环部分为苯氧基吡啶结构的 化合物(图 16). 其中, 化合物 49 在 $12.5 \mathrm{ppm}$ 下对二 斑叶螨有 $100 \%$ 的杀螨活性, 在 $500 \mathrm{ppm}$ 对小菜蛾具 有 $100 \%$ 的杀虫活性. 化合物 $\mathbf{5 0}$ 和 $\mathbf{5 1}$ 则在 $12.5 \mathrm{ppm}$ 下对小菜蛾具有 $100 \%$ 的杀虫活性，在 $500 \mathrm{ppm}$ 下对 二斑叶螨有 $100 \%$ 的杀螨活性.

\subsection{2 用苯氧乙胺基代替苄胺}

$\mathrm{B}$ 部分为苯氧乙胺基结构的化合物一般在苯环 的 2-位有甲基取代基，在 4-位有不同的氧乙基取代基.

1991 年, UBE Industries LTD 公司的 Obata 等 ${ }^{[19]}$ 报道了用苯氧乙胺基代替苄胺的一系列化合物(图 17), 化合物 52 在 $300 \mathrm{ppm}$ 下对二斑叶螨的成螨和螨 卵以及绿叶稻蝉有 $100 \%$ 的致死率，在 $500 \mathrm{ppm}$ 下对 小麦锈病和大麦白粉病有很好的抑制作用. 化合物 53 55 除了对大麦白粉病没有抑制作用外，对其他病 菌具有类似的活性.

1993 年, Obata 等 ${ }^{[20]}$ 又报道了吡唑环 3-, 4-位成环 的此类化合物(图 18). 化合物 $\mathbf{5 6}$ 和 $\mathbf{5 7}$ 在 $100 \mathrm{ppm}$ 下 对绿叶稻蝉, $300 \mathrm{ppm}$ 下对棕色叶蝉、二斑叶螨(成螨 和螨卵) 以及钻石背蛾有 $100 \%$ 的致死率, $500 \mathrm{ppm}$ 下 对大麦白粉病、小麦褐锈病、小麦锈病有很好的抑制 性. 化合物 $\mathbf{5 8}$ 在 $100 \mathrm{ppm}$ 下对绿叶稻蝉, $300 \mathrm{ppm}$ 下 对棕色叶蝉、二斑叶螨(成螨和螨卵)有 $100 \%$ 的致死

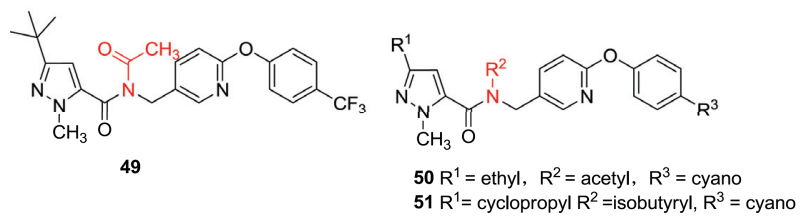

图 $16 \mathrm{~N}$ 酰基 $\mathrm{C}$ 环部分为苯氧基吡啶结构的化合物(网络版 彩图)

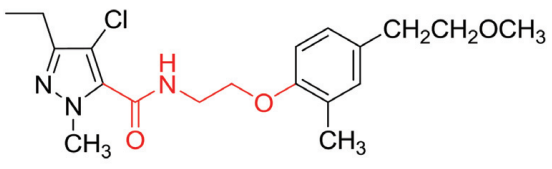

52

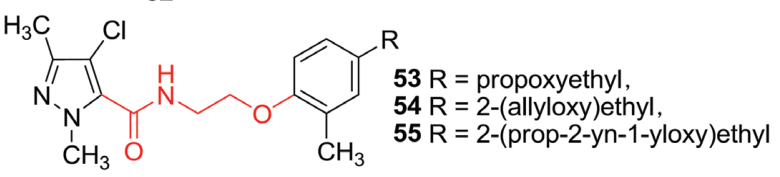

图 17 含有苯氧乙胺基结构的化合物(网络版彩图) 
<smiles>CCOCCc1ccc(OCCNC(=O)c2c3c(nn2C)C(C)CC3)c(C)c1</smiles>

56<smiles>C=CCOCCc1ccc(OCCNC(=O)c2c3c(nn2C)CCC3C)c(C)c1</smiles>

57<smiles>CCC(CC)CCc1ccc(OCCNC(=O)c2c3c(nn2C)C(C)CC3)c(C)c1</smiles>

图 18 吡唑环 3-, 4-位成环且含有苯氧乙胺基的化合物(网 络版彩图)

率, $500 \mathrm{ppm}$ 下对大麦白粉病、小麦褐锈病有很好的 抑制性.

UBE Industries 公司在 1994 年报道了 $\mathrm{N}$ 上有酰 基取代的此类化合物(图 19) ${ }^{[21]}$. 化合物主要表现杀 螨(二斑叶螨)活性. 其中化合物 $\mathbf{5 9}$ 在 $300 \mathrm{ppm}$ 对绿叶 稻蝉和二斑叶螨(成螨和螨卵)有 $100 \%$ 的致死率, 在 $500 \mathrm{ppm}$ 下对稻瘟病有 $100 \%$ 的抑制性. 化合物 60 在 $300 \mathrm{ppm}$ 对绿叶稻蝉和二斑叶螨(成螨和螨卵)有 100\%的致死率, 在 $500 \mathrm{ppm}$ 下对大麦白粉病有 $100 \%$ 的抑制性. 化合物 61 则主要表现杀螨活性.

在探究如何通过酰胺氮上的修饰提高化合物的<smiles>COCCc1ccc(OCCN(C(C)=O)C(=O)c2c(Br)c(C)nn2C)c(C)c1</smiles><smiles>CCOCCc1ccc(OCCN(C(=O)COC)C(=O)c2c(Cl)c(C)nn2C)c(C)c1</smiles><smiles>CCOCCc1ccc(OCCN(C(C)=O)C(=O)c2cc(C(C)(C)C)nn2C)c(C)c1</smiles>

图 $19 \mathrm{~N}$ 上有酰基取代的含有苯氧乙胺基的化合物(网络 版彩图)
杀螨活性同时降低化合物对鱼的毒性, Ube Industries 公司的 Obata 等 ${ }^{[22]}$ 研究了图 20 所示结构的化合物. 在研究中他们发现, 酰胺氮上具有酰基取代时, 化合 物活性提高的同时可以降低对鱼的毒性. 当 $R^{2}$ 为氯 时, 氮上进行酰基化对于提高活性和降低毒性均不 明显, 但当 $\mathrm{R}^{2}$ 为氢时, $\mathrm{N}$ 上酰基的引入可以明显降低 对鱼的毒性(表 3).

从表 3 中可以发现, $\mathrm{R}^{1}$ 为叔丁基时可以在提高杀 螨活性的同时降低对鱼的毒性. 随后, 固定吡唑环 3位为叔丁基, 研究酰胺氮上酰基的改变对杀螨的活 性和对鱼毒性的影响(表 4).

通过活性研究发现, 不同的 $\mathrm{R}^{3}$ 基团对化合物选 择性活性的影响不是特别明显.

2012 年, 南开大学汪清民教授课题组 ${ }^{[23]}$ 重点研 究了酰胺键部分对杀虫、杀螨活性的影响, 设计并合 成了酰胺键部分含有羟甲基和氯甲基的化合物以及 含有恶唑啉结构的骨架新颖的化合物(图 21).

生物活性测试结果表明, 这 3 类化合物对棉铃 虫、小菜蛾、蚜虫和蚊幼虫等表现出广谱的杀虫活性, 而且选择性地对棉铃虫表现出优异的杀虫活性. 其 中，如图 22 所示化合物 72 74 在 $5 \mathrm{mg} / \mathrm{kg}$ 下对棉铃虫 仍有 $60 \%$ 的致死率，活性水平与唑虫酰胺相当，同时 还发现这些衍生物对小菜蛾表现出拒食活性.

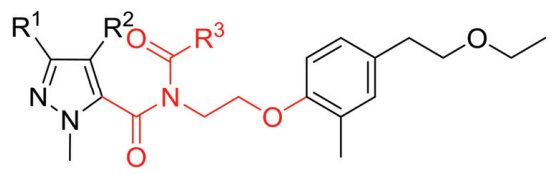

图 20 不同取代基的含有苯氧乙胺基的化合物(网络版彩图)

表 3 吡唑环-3-位取代基 $\mathrm{R}^{1}$ 对杀螨活性以及对鱼的毒性的 影响 ${ }^{\text {a) }}$

\begin{tabular}{cccccccc} 
& & & & & \\
\hline
\end{tabular}
率为 0 29\%、30\% 79\%、80\% 99\%和 100\%, 对鱼的毒性为 0 、 1\% 50\%、51\% 99\%和 100\%; b) 未进行测试 
表 4 酰胺氮上酰基化对化合物杀螨活性以及对鱼的毒性的 影响 a)

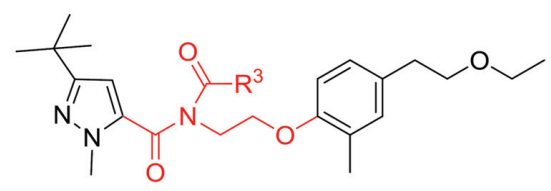

\begin{tabular}{ccccccccc}
\hline \multirow{2}{*}{ No. } & \multirow{2}{*}{$\mathrm{R}^{3}$} & \multicolumn{3}{c}{ 杀螨活性(ppm) } & & \multicolumn{2}{c}{ 毒性(ppm) } \\
\cline { 3 - 6 } \cline { 5 - 6 } & & 300 & 30 & 10 & 3 & & 0.5 & 0.05 \\
\hline $\mathbf{6 7}$ & $\mathrm{CH}_{3}$ & 3 & 3 & 2 & 0 & & 1 & 0 \\
$\mathbf{6 8}$ & $\mathrm{C}_{2} \mathrm{H}_{5}$ & 3 & 3 & 0 & 0 & 1 & 0 \\
$\mathbf{6 9}$ & iso- $\mathrm{C}_{3} \mathrm{H}_{7}$ & 3 & 3 & 3 & 0 & 0 & 0 \\
$\mathbf{7 0}$ & cyclo- $\mathrm{C}_{3} \mathrm{H}_{5}$ & 3 & 3 & 1 & 0 & 0 & 0 \\
$\mathbf{7 1}$ & Phenyl & 3 & 3 & 0 & - & 0 & 0 \\
\hline
\end{tabular}

a) 活性水平级别用 $0 \sim 3$ 表示, 分别对应对二斑叶螨的杀螨 率为 $0 \sim 29 \% 、 30 \% \sim 79 \% 、 80 \% \sim 99 \%$ 和 $100 \%$, 对鱼的毒性为 0 、 $1 \%$ 50\%、 $51 \%$ $99 \%$ 和 $100 \%$

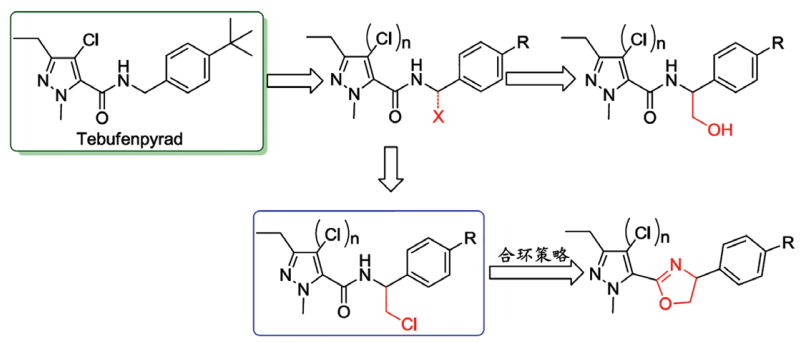

图 21 对酰胺键部分结构修饰(网络版彩图)<smiles>[Z20]C(NC(=O)c1cc(CC)nn1C)c1ccc(C(C)(C)C)cc1</smiles><smiles>CCc1nn(C)c(C2=NC(c3ccc(Cl)cc3)CO2)c1C1=NC(c2ccc(Oc3ccccc3)cc2)CO1</smiles>

图 22 部分高活性化合物(网络版彩图)

\section{3 对 $\mathrm{C}$ 部分的修饰}

\subsection{1 用其他芳香杂环代替苯环}

1990 年, 日本三菱化成株式会社的 Okada 等 ${ }^{[24]}$ 报道了具有如图 23 所示骨架结构的化合物. 其中化 合物 75 在 $50 \mathrm{ppm}$ 下对同翅目害虫褐飞風、鳞翅目害 虫小菜蛾、二斑叶螨以及稻瘟病具有 $100 \%$ 防治效果.
化合物 76 在 $50 \mathrm{ppm}$ 下对同翅目害虫褐飞虫、鳞翅目 害虫小菜蛾、二斑叶螨具有 $100 \%$ 防治效果. 化合物 77 在 $5 \mathrm{ppm}$ 下对小菜蛾的杀虫率达到 100\%. 化合物 78 在 $500 \mathrm{ppm}$ 下对二斑叶螨、褐飞虫、小菜蛾以及 稻瘟病有 $100 \%$ 的防治效果.

1993 年, 日本三菱化成株式会社 Natsume 等 ${ }^{[25]}$ 报道了用噻唑环代替苯环的一系列化合物(图 24). $\mathrm{R}^{4}$ 主要为烷氧基、对甲(硫)基苯氧基、氟代烷氧基. 其 中化合物 79 在 $500 \mathrm{ppm}$ 下对鳞翅目害虫小菜蛾、同 翅目褐飞風、鞘翅目害虫绿豆象和二斑叶螨以及小麦 白粉病、叶锈病有 $100 \%$ 的活性.

1990 年，日本三菱化成株式会社的 Yamada 等 ${ }^{[26]}$ 报道了同样用噻唑环代替苯环的一系列化合物(图 25). 化合物 80 82 在 $500 \mathrm{ppm}$ 下对小菜蛾、二斑叶

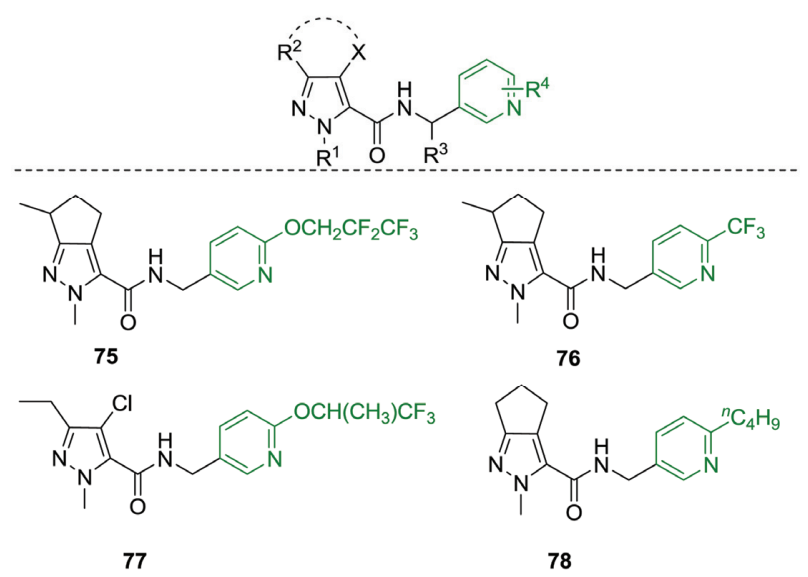

图 $23 \mathrm{C}$ 环为吡啶环结构的化合物(网络版彩图)<smiles>[R]c1nn([R])c(C(=O)NC([R])c2cnc([Y]([H])=[IH])s2)c1C(=O)NCc1nn(C)c(C(=O)NCc2cnc(OC(C)C)s2)c1Cl</smiles>

图 $24 \mathrm{C}$ 环为噻唑环结构的化合物(网络版彩图)

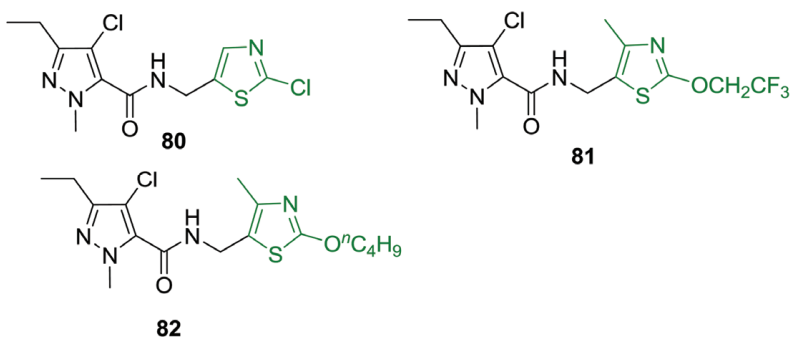

图 $25 \mathrm{C}$ 环为噻唑环结构的化合物 80 82 的结构式(网络版 彩图) 
螨以及小麦叶锈病具有一定的抑制活性.

2003 年, 日本农药公司的 Okada 等 ${ }^{[27]}$ 报道了 C 环 部分为苯并噻唑结构的一类化合物 (图 26), 其中化合 物 83 86 在 500 ppm 下对小麦白粉病有 100\%的活性.

\subsection{2 用二苯醚代替苯环}

1996 年, 日本三菱化学株式会社的 Okada 等 ${ }^{[28]}$ 设计合成了 $\mathrm{C}$ 环部分为二苯醚结构的化合物(图 27). 通过研究苯环对位的取代基 $\mathrm{R}$ 和吡唑环 3-, 4-位的取 代基对化合物杀虫活性的影响, 最终篎选出吡唑环 3-，4-位合环成五元环且在 6-位有甲基时活性最好. 扩环或改变甲基的位置以及把甲基换成乙基都会降 低其杀虫活性.

同时，他们发现 $\mathrm{R}$ 为吸电子基时化合物表现出 较好的杀虫活性, 当 $\mathrm{R}$ 为三氟甲基时活性最好, 在 $3.1 \mathrm{ppm}$ 下对同翅目害虫、鳞翅目害虫以及螨均表现 出优异的杀虫、杀螨活性.

1999 年, 日本三菱化学株式会社的 Okada 等 ${ }^{[29]}$ 设计合成了如图 28 所示结构的化合物, 重点研究了 吡唑环上的取代基以及两个苯环之间的连接方式对 活性的影响. 首先, 固定芐胺部分为 4-(4-甲基苯氧 基)苄胺, 研究吡唑环上的取代基 $\mathrm{R}$ 和 $\mathrm{X}$ 对化合物杀 小菜蛾和蚜虫活性的影响. 研究发现, 当 $\mathrm{R}$ 为乙基、 $\mathrm{X}$ 为氯时活性最好. 随后, 选取 $R$ 为乙基、 $X$ 为氯, 研究
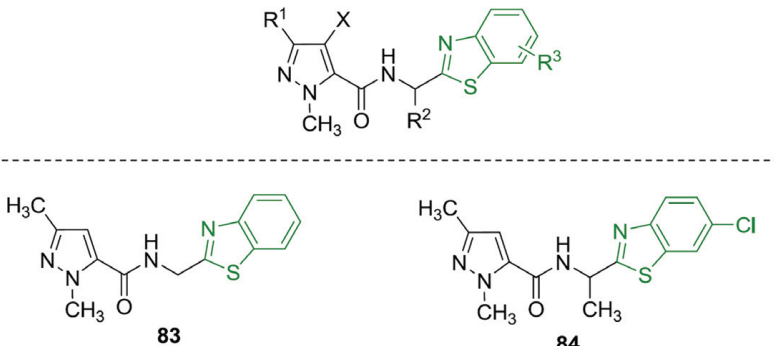

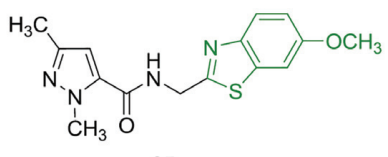

85

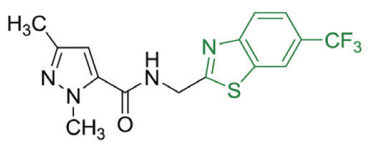

86
图 $26 \mathrm{C}$ 环部分为苯并噻唑结构的化合物(网络版彩图)

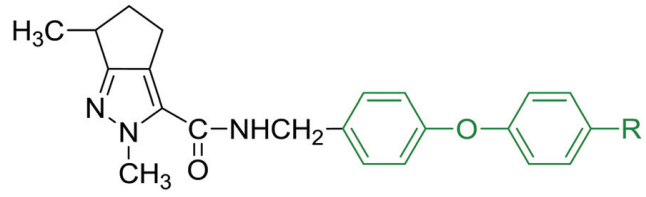

图 $27 \mathrm{C}$ 环部分为二苯醚结构的化合物(网络版彩图)
两个苯环之间的连接方式对活性的影响. 通过生物 活性测试发现, 当 $\mathrm{Y}$ 为氧原子且在两个苯环的对位 时活性最好即商品化品种唑虫酰胺.

1997 年, 日本三菱化学株式会社的 Kyomura 等 ${ }^{[30]}$ 报道了含有吡啶芳醚结构的一系列化合物(图 29). 此类化合物主要表现杀虫和杀菌作用，化合物 87 90 在 $500 \mathrm{ppm}$ 下对小菜蛾、褐飞虫具有 $100 \%$ 的 活性, $250 \mathrm{ppm}$ 下对稻瘟病和马铃薯晚疫病也具有 $100 \%$ 的活性.

1997 年, 三菱化学株式会社的 Okada 等 ${ }^{[31]}$ 设计 了 $\mathrm{C}$ 环为吡啶环的一类化合物, 结构如图 30 所示.

通过测试这些化合物对二斑叶螨的杀螨活性发 现，当 $\mathrm{R}$ 为烃基时活性顺序如下：叔丁基 $>$ 新戊基 $>$ 戊 基 $>$ 异丙基、丁基、异丁基 $>$ 丙基 $>$ 氢，说明当取代基 为烷基时，空间位阻大的取代基对化合物的杀螨活 性有利。当取代基换成烷氧基时，多氟取代的烷氧基 活性要优于没有氟取代的烷氧基. 其中, 2-, 2-, 3-, 3-, 4-, 4-, 4-取代的七氟丁氧基的杀螨活性与叔丁基的相 当, 但均低于吡螨胺的杀螨活性.

\subsection{3 用联苯型芳香环代替苯环}

具有此类结构的化合物具有一些共同的特点：在 吡唑环的 4-位一般为氢, 此类化合物主要表现杀菌

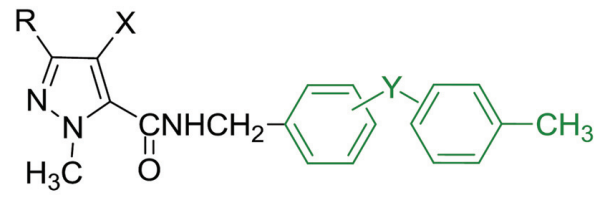

图 28 吡唑环上含有不同取代基且含有二苯梄结构的化合 物(网络版彩图)
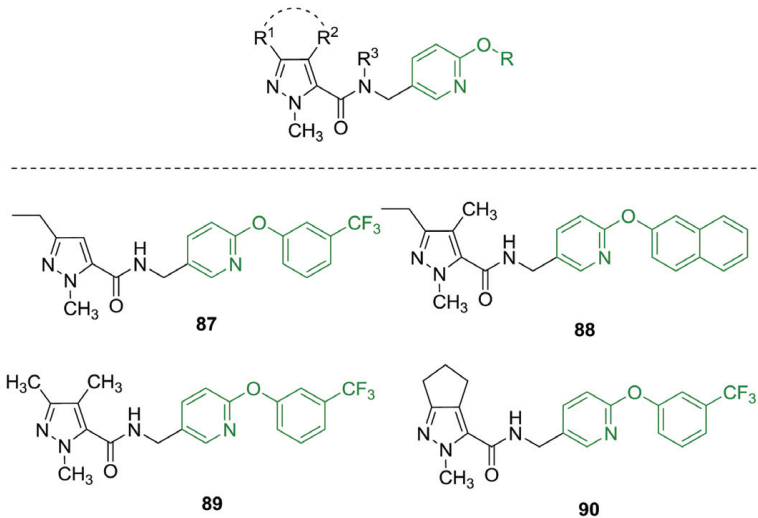

图 29 含有吡啶芳醚结构的化合物(网络版彩图) 
活性.

2002 年, 日本农药公司的 Okada 等 ${ }^{[32]}$ 报道了用 联苯来代替苯环的一系列化合物 (图 31). 其中化合物 91 94 在 $500 \mathrm{ppm}$ 下对小麦白粉病、小麦锈病、斜纹 夜蛾以及二斑叶螨活性均为 $100 \%$, 尤其是化合物 91 在 $0.8 \mathrm{ppm}$ 下对二㸚叶螨成螨有 $100 \%$ 的杀螨活性.

2003 年，同样是日本农药公司的 Takizawa 等 ${ }^{[33]}$ 报道了如图 32 所示结构的化合物. 化合物 95 和 96 在 $500 \mathrm{ppm}$ 下对小麦白粉病, $125 \mathrm{ppm}$ 下对小麦锈病 有 100\%的活性，化合物 97 和 98 只具有杀菌活性.

2005 年, 日本农药公司的 Takizawa 等 ${ }^{[34]}$ 报道了

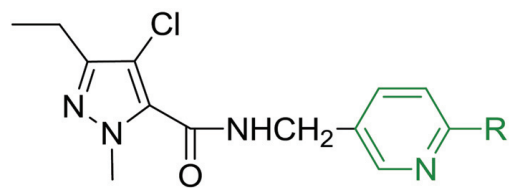

图 $30 \mathrm{C}$ 环为吡啶环的化合物(网络版彩图)
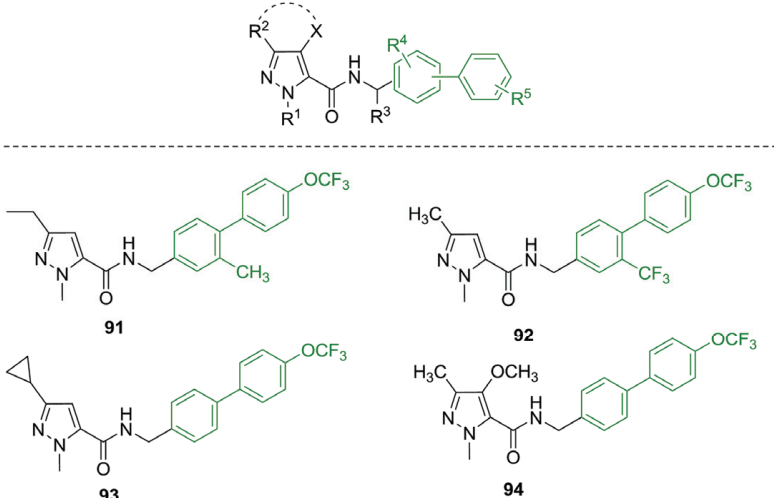

图 $31 \mathrm{C}$ 环部分为联苯结构的化合物(网络版彩图)
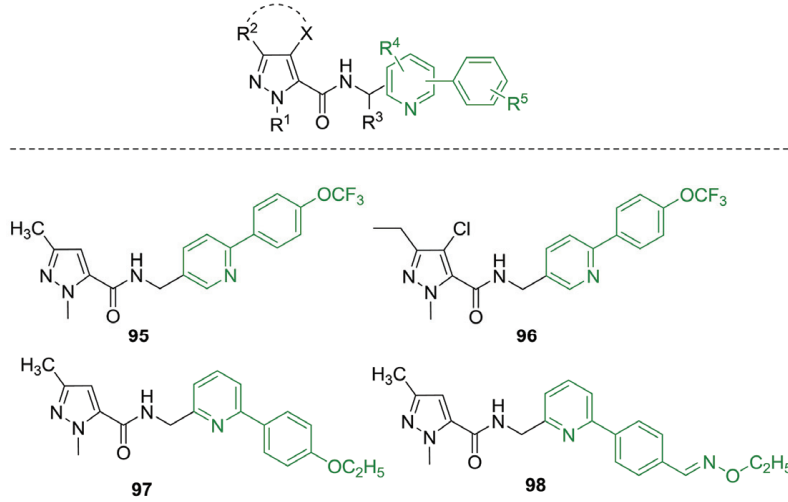

图 $32 \mathrm{C}$ 环部分为吡啶联苯结构的化合物(网络版彩图)
$\mathrm{C}$ 环部分为噻唑、吡唑等五元杂环与苯环相连的化合 物(图 33), 并对合成的化合物进行了杀菌、杀虫和杀 螨活性的研究.

2005 年, 国家南方农药创制中心江苏基地的徐 尚成等 ${ }^{[35}$ 报道苯联二恶唑结构的一类化合物(图 34). 化合物 104 和 105 在 $500 \mathrm{ppm}$ 下对粘虫、小菜蛾、䖯 豆蚜以及二斑叶螨有 $100 \%$ 的杀虫、杀螨活性，化合 物 104 在 $10 \mathrm{ppm}$ 下对䖯豆蚜仍有 100\%的杀虫活性.

\subsection{4 苯环上有多卤素取代基}

通过在苯环上引入卤素或含有卤素的取代基可 以明显提高化合物的杀虫活性. 1993 年, Bettarini 等 ${ }^{[36]}$ 报道了苯上连有多氟取代烷氧和烷硫基的一类化合 物(图 35). 化合物 106 109 在 $10 \mathrm{ppm}$ 下对马铃薯甲 虫成虫以及二斑叶螨有 $100 \%$ 的杀虫、杀螨活性.

1994 年, Bettarini 等 ${ }^{[37]}$ 报道了苯环上有多氟取代 以及 3-, 5-二氯取代的化合物(图 36). 其中化合物 110 在 $1 \mathrm{ppm}$ 下对二㸚叶螨成虫有 $100 \%$ 的杀螨活性.
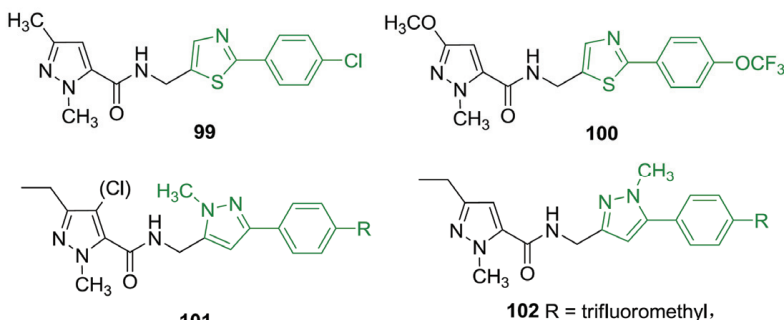
100

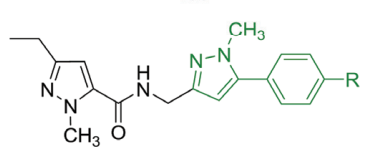

$102 \mathrm{R}=$ trifluoromethyl,

图 33 C 环部分为五元芳杂环联苯结构的化合物(网络版 彩图)

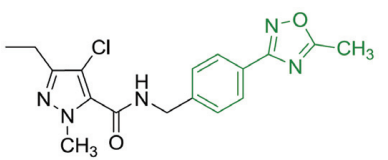

104

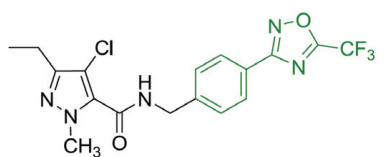

105
图 $34 \mathrm{C}$ 环部分为苯联二恶唑结构的化合物(网络版彩图)

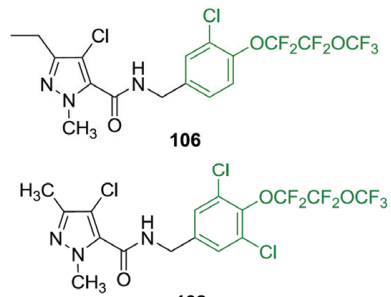

108

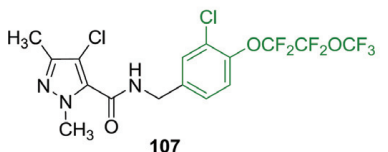

(c)

109
图 35 苯环上有多卤素取代的化合物(网络版彩图) 

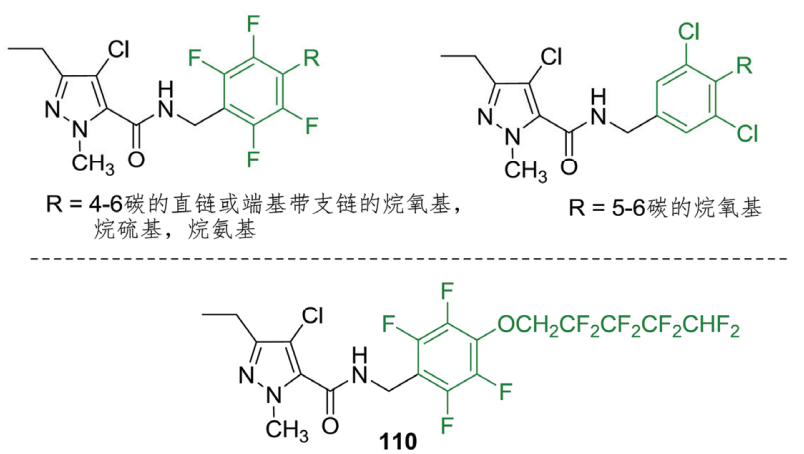

图 36 苯环上有多氟取代以及 3-, 5-二氯取代的化合物(网 络版彩图)

2013 年, 汪清民课题组 ${ }^{[38]}$ 基于线粒体呼吸作用 抑制剂的作用靶标复合物 I (complex I) 研究的最新进 展，借鉴文献中报道的构效关系，设计合成了苯环对 位含有亚胺、肜醚、肜酯和恶唑啉结构的化合物(图 37).

生物活性测试结果表明, 这些衍生物表现出广 谱的杀虫活性, 对棉铃虫、蚜虫、蚊幼虫以及朱砂叶 螨均表现出较好的活性. 其中, 化合物 111 (图 38)在 $11 \mathrm{mg} / \mathrm{kg}$ 的浓度下对棉铃虫有 $60 \%$ 的杀虫活性. 化合 物 112 和 113 在 $200 \mathrm{mg} / \mathrm{kg}$ 对蚜虫分别有 $100 \%$ 和 $90 \%$ 的杀虫活性. 含有二氢异恶唑啉结构的化合物 114 在 $1 \mathrm{mg} / \mathrm{kg}$ 对蚊幼虫有 $60 \%$ 的杀虫活性, 与吡螨胺的杀 虫活性相当. 该化合物在 $50 \mathrm{mg} / \mathrm{kg}$ 对朱砂叶螨的成 螨有 $60 \%$ 杀螨活性.

\section{3 吡唑-5-甲酰胺类杀虫、杀螨剂构效关系}

\section{1 对 $\mathrm{A}$ 部分的修饰}

对 A 部分的修饰主要是对吡唑环 3-, 4-位的修饰, 已有研究结果表明, 吡唑环上的取代基空间位阻不

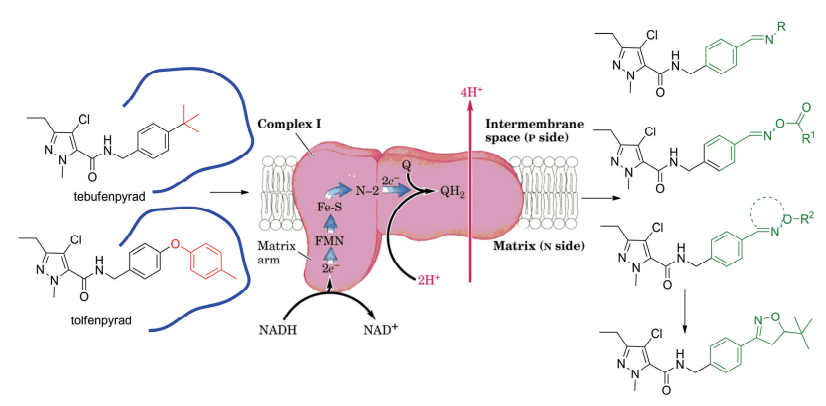

图 37 基于受体复合物 I 的化合物的设计(网络版彩图)

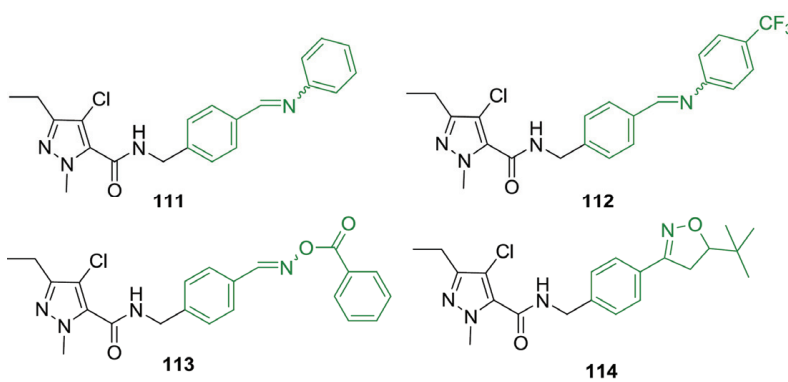

图 38 部分高活性化合物(网络版彩图)

能太大, 主要是一些 2 3 个碳的取代基. 按结构来分 大体上可以分为以下几类: (1) 让 3-, 4-位成环, 具有 此类结构的化合物在杀虫、杀螨活性方面有不错的表 现. 例如, 化合物 6 对二斑叶螨和相橘全爪螨成螨和 螨卵的活性要优于吡螨胺(图 39); 化合物 115 在 $3 \mathrm{ppm}$ 下对黑尾叶蝉、稻飞䖝和桃蚜表现出优异的杀 虫活性(图 39). (2) 4-位的取代基如成烷氧基、酯基、 不饱和取代基等, 该类化合物除了具有杀螨活性外, 还具有杀菌活性. (3) 用其他杂环代替吡唑环, 主要 是用吲唑环代替吡唑环, 具有此类结构的化合物主 要表现出杀菌活性.

\section{2 对 $B$ 部分的修饰}

对 $\mathrm{B}$ 部分的修饰主要有两类: 在酰胺 $\mathrm{N}$ 上进行 酰基化, 主要是为了调节化合物的溶解性以及增加 可与靶标结合的位点. 用苯氧乙胺基代替苄胺, 具有 此类结构的化合物对绿叶稻蝉、棕色叶蝉、二斑叶螨 (成螨和螨卵)以及钻石背蛾、大麦白粉病、小麦褐锈 病有很好的抑制性.

\section{3 对 $\mathrm{C}$ 部分的修饰}

已有的构效关系表明，苯环对位空阻较大的疏

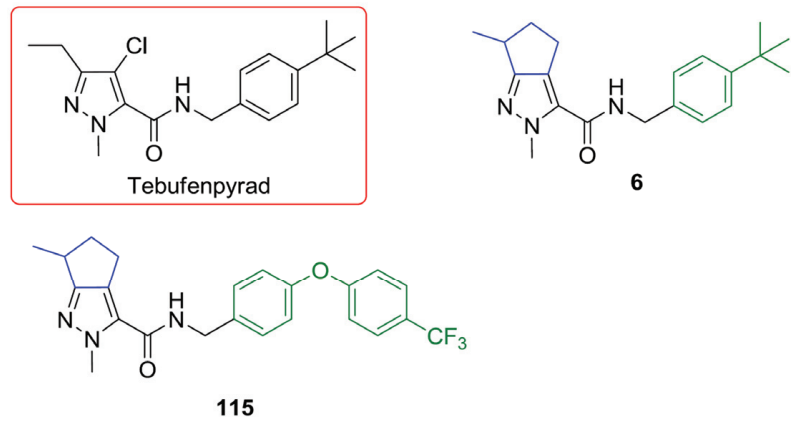

图 39 对 $\mathrm{A}$ 部分的修饰(网络版彩图) 
水性取代基是分子具有较高杀螨活性的重要条件; 烷氧基尤其是氟代烷氧基, 以及苯环对位有 $\mathrm{CF}_{3}$ 、 $\mathrm{OCF}_{3} 、 \mathrm{SCH}_{3}$ 取代的苯氧基时表现出较高的杀虫活性. 对苯环部分的修饰是吡唑-5-甲酰胺类杀虫、杀螨剂 研究的重点, 大体可以分为: (1) 用其他芳香杂环代 替苯环. 用吡啶环代替苯环时, 吡啶环上的取代基一 般为氟代烷氧基和苯氧基. 当苯环对位的取代基为 苯氧基时, 苯氧基对位一般有 $\mathrm{CF}_{3} 、 \mathrm{SCH}_{3} 、 \mathrm{OCF}_{3}$ 等 取代基时活性优于其他取代基. (2) 用联苯型芳香环 代替苯环. 联苯型主要表现杀菌活性, 但个别化合物 表现出优异的杀螨活性. 例如, 化合物 91 在 $0.8 \mathrm{ppm}$ 下对二斑叶螨成螨有 100\%的杀螨活性(图 40). (3) 在 苯环上进行多卤素取代. 苯环上有卤素取代时在杀 螨活性基本保持不变的情况下, 杀虫活性得到显著 提高. 化合物 110 对二斑叶螨成螨在 $1 \mathrm{ppm}$ 下有

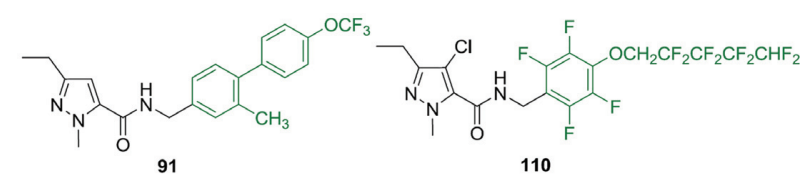

图 40 对 $\mathrm{C}$ 部分的修饰(网络版彩图)

$100 \%$ 的杀螨活性(图 40).

\section{4 结论}

本文按照结构的不同综述了 $N$-烷基吡唑-5-甲酰 胺类杀虫、杀螨剂的发展概况. 自吡螨胺被报道以来, 对于此类具有独特作用机理的杀虫、杀螨剂的研究备 受关注, 是近年来杀虫、杀螨剂研究的热点. 预计 $N$ 烷基吡唑-5-甲酰胺类杀虫、杀螨剂在未来将具有更 加广阔的前景.

\section{参考文献}

1 Prabhaker N, Toscano CN, Castle JS, Henneberry T. J Pestic Sci, 1997, 51: 419-428

2 Matsumura M, Takeuchi H, Satoh M, Sanada-Morimura S, Otuka A, Watanabe T, Thanh DV. Pest Manag Sci, 2008, 64: 1115-1121

3 柴宝山, 刘远雄, 杨吉春, 刘长令. 农药, 2007, 46: 800-809

4 郎玉成, 柏亚罗. 吡唑类农药品种的研究开发进展. 现代农药, 2006, 5: 6-12

5 Okada I, Okui S, Takahashi Y, Fukuchi T. J Pestic Sci, 1991, 16: 623-629

6 Okada I, Okui S, Nishimata M, Takahashi Y, Fukuchi T. Pyrazolecarboxamides as insecticides and miticides and their preparation. European Patent, 307801A1, 1989-03-22

7 Okada I, Okui S, Sekine M, Takahashi Y, Fukuchi T. J Pestic Sci, 1992, 17: 69-73

8 Farooq S, Hall RG, Ciba-Geigy AG. Indazole derivatives. WO Patent, 9405642A1, 1994-03-17

9 Kyomura N, Okui S, Ikeda Y, Suzuki S, Tomita H, Higashino Y. Preparation of (pyridylmethylaminocarbonyl) indazoles as fungicides, insecticides and miticides. European Patent, 726266A1, 1996-08-14

10 Okada I, Suzuki S, Takahashi Y. Preparation of pyrazole derivatives as insecticides and acaricides. Japan Patent, 02264760A, 1990-10-29

11 Tanaka T, Okui S, Fukuchi T. Preparation of alkoxypyrazolecarboxamide as insecticides, acaricides, and agricultural and horticultural fungicides. Japan Patent, 07173139A, 1995-07-11

12 Okimura N, Ikeda Y, Okui K. Preparation of pyrazoles, and agrochemical microbicides, insecticides, and acaricides containing them. Japan Patent, 10053583A, 1998-02-24

13 Kano H, Ikeda Y, Kyomura N, Tomita H, Fukuchi T. Preparation of pyrazolecarboxamides as insecticides, acaricides, and fungicides. WO Patent, 9946247A1, 1999-09-16

14 Okada I, Okui S, Takahashi T, Hosokawa A, Kyomura N, Fukachi T, Takahashi Y. J Pestic Sci, 1994, 19: 317-320

15 Tanaka T, Suzuki S, Takahashi Y. Preparation of $N$-acyl-5-pyrazolecarboxamides as insecticides and acaricides. Japan Patent, 03068560A, 1991-3-25

16 Shiga Y, Okada I, Ikeda Y, Takizawa E, Fukuchi T. J Pestic Sci, 2003, 28: 313-314

17 Okada I, Ikeda Y. Preparation of pyrazolecarboxylic acid amides and insecticides and acaricides containing them. Japan Patent, 2001172261A, 2001-06-26

18 Okada I, Ikeda Y. Preparation of pyrazole-5-carboxamides and their use for insecticides and acaricides. Japan Patent, 2001199984A, 2001-07-24

19 Obata T, Fujii K, Tanaka T, Ooka A, Shikita S. Preparation of 5-( $N$-phenoxyalkylcarbamoyl) pyrazoles as insecticides, acaricides, and fungicides. European Patent, 405808A1, 1991-01-02

20 Obata T, Fujii K, Ooka A, Shikita S. Preparation of cyclopenta [1,2-c] pyrazolecarbox-amides as pesticides. European Patent, 521409A1, 1993-01-07 
21 Obata T, Ooka A, Fujii K, Suizu S. Preparation of insecticidal, acaricidal and fungicidal $N$-(phenoxyalkyl)pyrazolecarboxamides. European Patent, 618195A1, 1994-10-05

22 Obata T, Fujii K, Funaki E, Tsutsumiuti K, Ohoka A, Suizu S, Kanetsuki Y. J Pestic Sci, 1999, 24: 33-37

23 Song HJ, Liu YX, Xiong LX, Li YQ, Yang N, Wang QM. J Agric Food Chem, 2012, 60: 1470-1479

24 Okada I, Suzuki S, Okui S, Takahashi Y, Fukuchi T, Nakajima T. Preparation of [(5-pyrazolylcarboxamido) alkyl]pyridine derivatives and insecticidal, miticidal, and fungicidal compositions containing them as active ingredients. European Patent, 329020A1, 1989-08-23

25 Natsume B, Kyomura N, Kikutake K, Fukuchi T. Preparation of pyrazolecarboxamides as pesticides. European Patent, 462573A1, 1991-12-27

26 Yamada M, Okui S, Okimura N. Preparation of pyrazolecarboxamide derivatives as insecticides, acaricides, and agrochemical fungicides. Japan Patent, 05255268A, 1993-10-5

27 Okada I, Kikutake K. Preparation of $N$-(benzothiazolylmethyl)pyrazolecarboxamides and agrochemical fungicides containing them. Japan Patent, 2003160584A, 2003-6-3

28 Okada I, Okui S, Wada M, Takahashi Y. J Pestic Sci, 1996, 21: 305-310

29 Okada I, Okui S, Fukuchi T, Yoshiya K. J Pestic Sci, 1996, 24: 393-396

30 Kyomura N, Ikeda Y, Okui S, Tomita H, Higashino Y, Koike S. Preparation of pyrazole derivatives as agrochemical fungicides, insecticides, and acaricides. WO Patent, 9737990A1, 1997-10-16

31 Okada I, Suzuki S, Okui S, Fukuchi T, Takahashi Y. J Pestic Sci, 1997, 22: 230-232

32 Okada I, Takizawa E, Kikutake K, Fukuchi T. Preparation of pyrazolecarboxamide derivatives as pest control agents, fungicides, and acaricides. WO Patent, 2002083647A1, 2002-10-24

33 Takizawa E, Okada I, Kikutake K, Fukuchi T. Preparation of pyrazolecarboxamides as pesticides. WO Patent, 2003062222A1, 2003-07-31

34 Takizawa E, Okada I, Kikutake K. Carboxamide compounds and pest control agent containing the samen as active ingredient. Japan Patent, 200560255A, 2005-03-10

35 徐尚成, 倪珏萍, 郭丽琴, 马海军, 王风云, 刀亚梅, 万琴. 吡唑酰胺类化合物及其中间体和以该类化合物为活性成分的有害生物 防治剂。中国专利, CN1927860A, 2007-03-14

36 Bettarini F, Capuzzi L, La Porta P, Fukuchi T. Pyrazolecarboxamides exhibiting insecticidal and acaricidal activity. European Patent, 590720A1, 1991-12-27

37 Bettarini F, Meazza G, La Porta P, Zanardi G, Massimini S, Reggiori F. Preparation of (polyhalobenzylcarbamoyl) pyrazoles as acaricides and insecticides. European Patent, 634403A1, 1995-01-18

38 Song HJ, Liu YX, Xiong LX, Li YQ, Yang N, Wang QM. J Agric Food Chem, 2013, 61: 8730-8736

\title{
Recent advances in insecticides/acaricides of $N$-alkylpyrazole-5- formamides
}

\author{
Hongjian Song ${ }^{1}$, Yuxiu Liu ${ }^{1}$, Qingmin Wang ${ }^{1,2^{*}}$ \\ ${ }^{1}$ State Key Laboratory of Elemento-Organic Chemistry, Research Institute of Elemento-Organic Chemistry, Nankai University, Tianjin 300071, \\ China \\ ${ }^{2}$ Collaborative Innovation Center of Chemical Science and Engineering (Tianjin), Tianjin 300071, China \\ *Corresponding author (email: wangqm@nankai.edu.cn.)
}

\begin{abstract}
Insecticidal/acaricidal $N$-alkylpyrazole-5-formamides have attracted much attention because of their fine characteristics, such as high efficiency, low toxicity and unique mechanism. Meanwhile the researches on their synthesis and bioactivities have been important directions in the pesticide field. The recent advances of this type of compounds were reviewed by being classified according to their structures, and the structure-activity relationships were summarized.
\end{abstract}

Keywords: $N$-alkylpyrazole-5-formamides, tebufenpyrad, tolfenpyrad, insecticidal activity, acaricidal activity

doi: $10.1360 / \mathrm{N} 032016-00080$ 